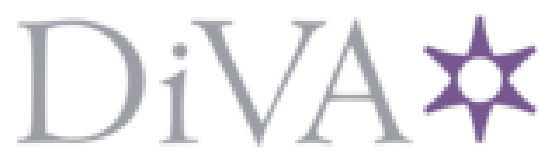

http://www.diva-portal.org

\title{
Postprint
}

This is the accepted version of a paper presented at 25th IEEE $\mid R S J$ International Conference on Intelligent Robots and Systems (IROS), OCT 07-12, 2012, Algarve, Portugal.

Citation for the original published paper:

La Hera, P., Rehman, B., Morales, D. (2012)

Electro-hydraulically actuated forestry manipulator: Modeling and Identification.

In: 2012 IEEE/RSJ Iinternational Conference on Intelligent Robots and Systems (IROS) (pp.

3399-3404). New York: IEEE conference proceedings

IEEE International Conference on Intelligent Robots and Systems

http://dx.doi.org/10.1109/IROS.2012.6385656

N.B. When citing this work, cite the original published paper.

Permanent link to this version:

http://urn.kb.se/resolve?urn=urn:nbn:se:umu:diva-727 17 


\section{Electro-hydraulically actuated forestry manipulator: Modeling and Identification}

\author{
Pedro La Hera \\ Swedish University of Agricultural Sciences \\ Umeå, Sweden, 90183 \\ Email: xavier.lahera@slu.se
}

\author{
Bilal Ur Rehman and Daniel Ortíz Morales \\ Department of Applied Physics and Electronics \\ Umeå University, Sweden, 90187 \\ Email(s): r3hman@gmail.com \\ daniel.ortiz.morales@tfe.umu.se
}

\begin{abstract}
We present results of modeling dynamics of a forestry manipulator, in which we consider its mechanics, as well as its hydraulic actuation system. The mathematical model of its mechanics is formulated by Euler-Lagrange equations, for which the addition of friction forces is straightforward. Dynamics of the hydraulic system is modeled upon first principle laws, which concern flow through orifices and fluid compressibility. These models lead to a set of equations with various unknown parameters, which are related to the inertias, masses, location of center of masses, friction forces, and valve coefficients. The numerical values of these parameters are estimated by the use of least-square methods, which is made feasible by transforming the models into linear representations. The results of simulation tests show a significant correspondence between measured and estimated variables, validating our modeling and identification approach.
\end{abstract}

\section{INTRODUCTION}

Modern forestry operations use state-of-art systems and high-tech machinery to meet the mechanical and engineering challenges of harvesting and logging trees in a safe and environmentally responsible manner. The cranes of these machines are an especial type of mechanical manipulators, which employ hydraulic actuation to produce motions. They are engineered to be manually maneuvered by joysticks, having the human operator as the control unit. During daily work, the driver is demanded to perform various tasks simultaneously, which include visualization, recognition, selection, controlling the crane, and positioning the vehicle. This level of work demand is stressful, since the operator has to deal with an excess of information, and take various decisions at high pace.

As an attempt to support human drivers, and in view of increasing the machine productivity [1], forestry based industry and researchers have analyzed the possibility to automate the routine motions performed in these tasks [2], [3]. The forwarder family of cranes used for logging has been established as a benchmark platform to understand the fundamental challenges of designing this (semi) automatic solution. From the industrial point of view, crane manufacturers have entrepreneur the design of forwarder cranes that can support the implementation of feedback control systems [4]. Researchers, on the other hand, have provided different solutions regarding automatic control design, study of human crane operation, and trajectory planning techniques. Many of these concepts have been experimentally validated in modern laboratories dedicated to the development of future forestry technologies [5], [6], [7], [8].

Formally, forwarder cranes can be regarded as electrohydraulically actuated mechanical systems. Theoretically, control design for such systems has proven to be challenging, due to the complex nonlinear nature of the process dynamics. In literature, we recognize the establishment of two well defined brands of development for these systems. On one hand, there exist a vast number of publications with quite mature content regarding modeling and controller design for hydraulic servo systems. Such terminology is attributed to the interconnection between valve and cylinder (or rotator), and the main objective is to control the actuator dynamics, e.g. [9], [10], [11], [12], [13], [14]. On the other hand, we have hydraulic manipulators, for which mainly modelfree controllers, resembling conventional decentralized joint control, are found [15], [16], [17], [6], [8], [18], [7]. This class of controllers are usually desirable due to their simplicity of implementation. However, experimental studies reveal that their efficiency is limited to joint trajectories with slow velocity profiles [17]. As the speed of motion reaches high (human) profiles, or when the payload changes dramatically, they lack of damping capabilities and stability.

To attain improving performance, it is of interest to apply model-based motion control strategies, employing models of robot kinematics and dynamics [19]. Within this context, modeling and parameters identification are key elements to realize such a design. Therefore, the initial interest is to verify that, despite the complex mechanical structure and hydraulic hardware, standard engineering procedures for robot modeling and parameter estimation are applicable for describing and simulating dynamics of these machines. The following presentation can be regarded as a technical report of results along these lines, which presents a detailed review of our experience modeling dynamics of the mechanics of a forwarder crane and its experimental validation.

\section{MODELING}

The manipulator used for our study is a downsized version of a typical forwarder crane ${ }^{1}$, but similar in configuration and

\footnotetext{
${ }^{1}$ All dimensional parameters required in all the coming set of equations are reported in [15].
} 
dynamics (see Fig. 1). This machine has been fully equipped to realize various experimental studies, as reported in [20].

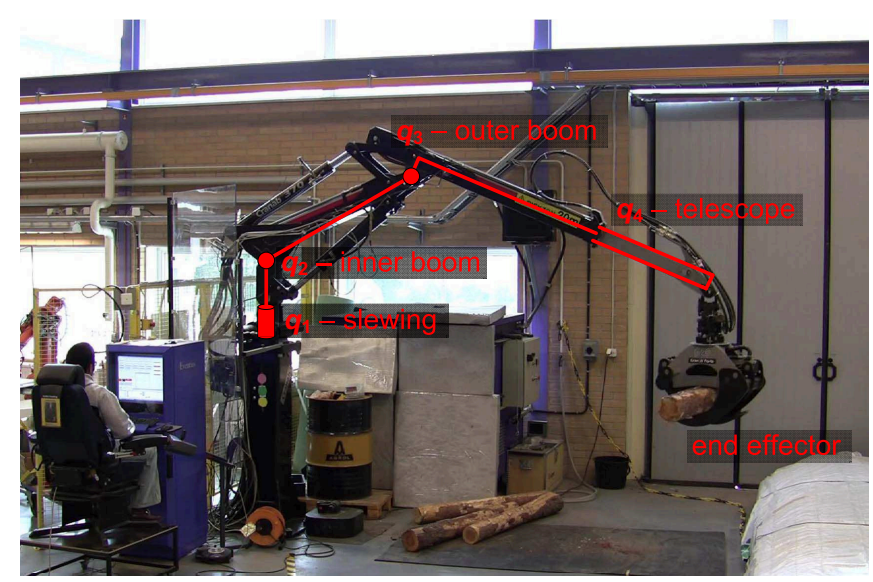

Fig. 1. Laboratory crane installed at the Department of Applied Physics and Electronics, Umeå University.

\section{A. Dynamics modeling}

We apply the Euler-Lagrange equation as a formalism used to systematically describe the robot dynamics [19], [21], [22]. Based on such a procedure, the computation of the Euler Lagrange equations of motion leads to a second order set of differential equations of the form

$$
M(q) \ddot{q}+C(q, \dot{q}) \dot{q}+G(q)=B(q) \tau,
$$

where $M(q)$ denotes a symmetric and positive-definite matrix of inertias, $G(q)$ the gravity vector, $C(q, \dot{q})$ the matrix of Coriolis forces, and $B(q)$ is a matrix that allocates the external forces to joint torques.

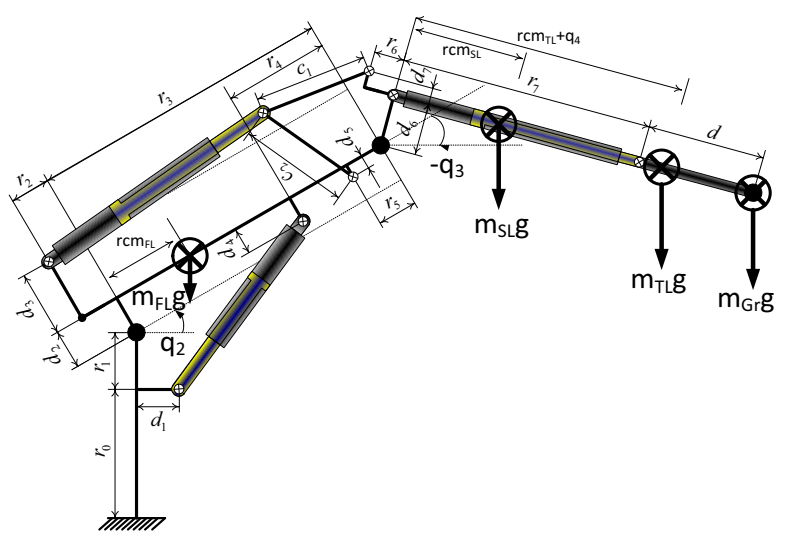

Fig. 2. Crane dimensions and masses description in the sagittal plane.

Considering motions in the plane, as shown in the schematics of Fig. 2, the model (1) takes the form

$M\left(q_{i}, \xi\right)\left[\begin{array}{c}\ddot{q}_{2} \\ \ddot{q}_{3} \\ \ddot{q}_{4}\end{array}\right]+C\left(q_{i}, \dot{q}_{i}, \xi\right)\left[\begin{array}{c}\dot{q}_{2} \\ \dot{q}_{3} \\ \dot{q}_{4}\end{array}\right]+G\left(q_{i}, \xi\right)=\left[\begin{array}{c}\tau_{2} \\ \tau_{3} \\ F_{4}\end{array}\right]$ where $q=\left[q_{2}, q_{3}, q_{4}\right]^{T}$, i.e. the slewing angle $q_{1}$ is not considered, and $\xi$ represents the crane inertial parameters. In addition, and assuming no frictional forces, the set of equations in (2) can be transformed and represented linearly in the elements of the base parameters $\xi$ that constitutes the minimum set [19], i.e.

$$
\varphi(q(t), \dot{q}(t), \ddot{q}(t)) \cdot \theta=\tau,
$$

where $\varphi(\cdot)$ denotes the regressor written in terms of the measurable variables $[q, \dot{q}, \ddot{q}], \theta=\left[\theta_{1}, \ldots, \theta_{7}\right]^{T}$ the minimum set of inertial parameters, which components are

$$
\begin{aligned}
\theta_{1}= & m_{F L} \cdot r c m_{F L}^{2}+I z z_{F L} \\
\theta_{2}= & r c m_{S L} \cdot m_{S L} \\
\theta_{3}= & m_{S L} \\
\theta_{4}= & m_{G r} \cdot r_{7}+r c m_{T L} \cdot m_{T L} \\
\theta_{5}= & m_{T L}+m_{G r} \\
\theta_{6}= & m_{G r} \cdot r_{7}^{2}+m_{T L} \cdot r c m_{T L}^{2}+\ldots \\
& I z z_{S L}+I z z_{T L}+m_{S L} \cdot r c m_{S L}^{2}+I z z_{G r} \\
\theta_{7}= & m_{F L} \cdot r c m_{F L},
\end{aligned}
$$

and $\tau$ the vector of generalized input torques and forces, which is equal to the right hand side of (2).

\section{B. Modeling friction forces}

A model often used to represent friction forces considers the static Coulomb - viscous friction, and it is formulated as

$$
\tau_{i}^{F}=f_{c, i} \cdot\left(\dot{q}_{i}\right)+f_{v, i} \cdot \dot{q}_{i},
$$

where $i=\{2,3,4\}$, and corresponds to the $i$ th link of the robot, $f_{v}$ denotes the viscous friction, and $f_{c}$ the Coulomb friction [23]. Hydraulic systems exhibit appreciable asymmetric friction forces, i.e. the constant parameters used to represent friction change according to the direction of motion, i.e.

$$
\begin{aligned}
& f_{v}=\bar{f}_{v}+\Delta f_{v} \cdot(\dot{q}), \\
& f_{c}=\bar{f}_{c}+\Delta f_{c} \cdot(\dot{q}),
\end{aligned}
$$

where the terms with a barr denote the mean values, and their variational values $\Delta$ depend on the direction of motion given by the $(\dot{q})$. Introducing the above equations into the general form (11) yields a more complete model for friction forces:

$$
\tau_{i}^{F}=\bar{f}_{c, i} \cdot\left(\dot{q}_{i}\right)+\Delta f_{c, i}+\bar{f}_{v, i} \cdot \dot{q}_{i}+\Delta f_{v, i} \cdot\left|\dot{q}_{i}\right|,
$$

which is able to capture the most relevant effects of the nonlinear friction phenomena.

\section{Combining the models of dynamics and friction forces}

Due to the linearity of the friction parameters in (14), i.e.

$$
\tau_{i}^{F}=\underbrace{\left[\begin{array}{llll}
\left(\dot{q}_{i}\right) & 1 & \dot{q}_{i} & \left|\dot{q}_{i}\right|
\end{array}\right]}_{\varphi_{i}^{f}} \cdot \underbrace{\left[\begin{array}{c}
\bar{f}_{c, i} \\
\Delta f_{c, i} \\
\bar{f}_{v, i} \\
\Delta f_{v, i}
\end{array}\right]}_{\theta_{i}^{f}},
$$


the introduction of frictional forces into the linear model (3) is straightforward. To show this, we form a complete set of friction forces for the three links considered, i.e. $q=$ $\left[q_{2}, q_{3}, q_{4}\right]^{T}$, as follows

$$
\tau^{F}=\underbrace{\left[\begin{array}{ccc}
\varphi_{2}^{f} & 0 & 0 \\
0 & \varphi_{3}^{f} & 0 \\
0 & 0 & \varphi_{4}^{f}
\end{array}\right]}_{\varphi^{f}} \cdot \underbrace{\left[\begin{array}{c}
\theta_{2}^{f} \\
\theta_{3}^{f} \\
\theta_{4}^{f}
\end{array}\right]}_{\theta_{f}},
$$

where the zero vectors have dimension 1-by-4. Thus, considering that frictions forces act opposed to the torques in the right hand side of (3), a more complete linear model is given by

$$
\left[\varphi(q(t), \dot{q}(t), \ddot{q}(t)) \quad \varphi_{f}(\dot{q}(t))\right] \cdot\left[\begin{array}{c}
\theta \\
\theta_{f}
\end{array}\right]=\tau,
$$

which results by the combination of (3) and (16).

\section{Mapping cylinder forces to joint torques}

Using the geometry of the machine, the mapping between the motion of the hydraulic cylinders given angles of the joints can be explicitly found. This mapping $x_{i}=f\left(q_{i}\right)$, can be used for $a$ ) deriving the joint torques, and $b$ ) computing the velocity of the pistons given angular velocity. To this end, we consider the virtual work principle [19], to define that

$$
\tau_{i} \cdot d q_{i}=F_{i} \cdot d x_{i}
$$

which yields an equality for the joint torque as

$$
\tau_{i}=F_{i} \cdot \frac{d x_{i}}{d q_{i}}
$$

for the links $i=2,3$, as required in $(2)^{2}$. The actuators forces (see Fig. 3) can be calculated as [13]

$$
F_{i}=A_{A, i} p_{A, i}-A_{B, i} p_{B, i}, \quad i=\{2,3,4\},
$$

where $A_{A, i}$ and $A_{B, i}$ denote the areas of chambers $A$ and $B$ respectively, and $p_{(\cdot)}$ the measurements of their corresponding pressures, which in the machine are available through pressure transducers.

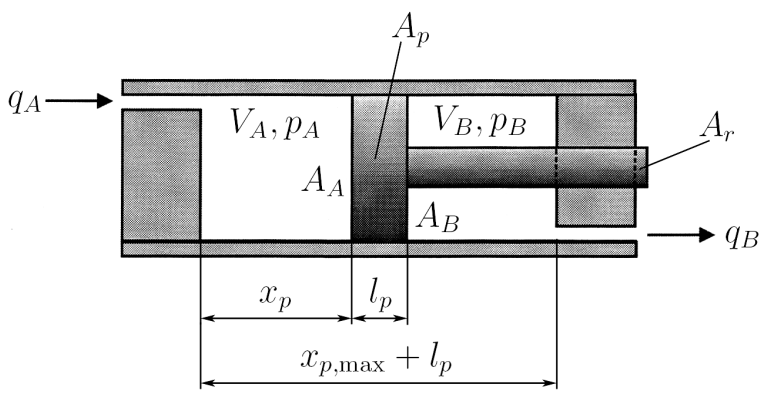

Fig. 3. Hydraulic single-rod cylinder [13].

\footnotetext{
${ }^{2}$ Recall that the telescopic displacement $q_{4}$ is linear, and therefore only its cylinder force $F_{4}$ is needed in the dynamics (2)
}

\section{E. Modeling the hydraulic actuation system}

In this machine, the hydraulic servo system consists of a directional proportional control valve, and differential single rod cylinders used as actuators to produce forces. Each piston's motion can be controlled by regulating the oil flow rates $Q_{A, i}$ and $Q_{B, i}$ in each chamber, which mathematical approximation is given by [13]:

$$
\begin{aligned}
& \left.\begin{array}{l}
Q_{A, i}=c_{1} u_{i} \sqrt{P_{s}-P_{A, i}} \\
Q_{B, i}=-c_{2} u_{i} \sqrt{P_{B, i}-P_{r}}
\end{array}\right\} u_{i} \geq 0 \\
& \left.\begin{array}{l}
Q_{A, i}=c_{3} u_{i} \sqrt{P_{A, i}-P_{r}} \\
Q_{B, i}=-c_{4} u_{i} \sqrt{P_{s}-P_{B, i}}
\end{array}\right\} u_{i}<0
\end{aligned}
$$

where $i=\{2,3,4\}$, and corresponds to either of the links, and $u_{i}$ is the electrical control input. $P_{s}$ and $P_{r}$ are the supplied and return pressures respectively, while $P_{A, i}$ and $P_{B, i}$ are the chamber pressures in the hydraulic cylinders, as shown in Fig. 3. The valve coefficients are denoted by $c_{1}, c_{2}$, $c_{3}$ and $c_{4}$, and they are related to the physical properties of the valve, which numerical values are usually not provided by the manufactures. The hydraulic pressures at both chambers can be defined by the differential equations:

$$
\begin{aligned}
& \dot{P}_{A, i}=\frac{\beta}{\left(V_{h_{A}, i}+A_{A, i} x_{p, i}\right)}\left(Q_{A, i}-\dot{x}_{p, i} A_{A, i}\right) \\
& \dot{P}_{B, i}=\frac{\beta}{\left(V_{h_{B}, i}+A_{B, i}\left(s_{i}-x_{p, i}\right)\right)}\left(Q_{B, i}+\dot{x}_{p, i} A_{B, i}\right),
\end{aligned}
$$

with $V_{h_{A}, i}$ and $V_{h_{B}, i}$ being the volumes of oil on each chamber when the cylinder is fully closed, $\beta$ the fluid Bulk modulus, $s_{i}$ the cylinder's maximum length, and $x_{p, i}$ the piston position, which is calculated according to:

$$
x_{p, i}=\left(x_{i}-x_{m, i}\right),
$$

where $x_{i}$ is the current length of the hydraulic cylinder, and $x_{m, i}$ its minimum length. The linear displacements of the cylinders are derived in [20], from which the piston's linear velocity can be calculated $\mathrm{as}^{3}$ :

$$
\dot{x}_{p, i}=\dot{x}_{i}=\frac{\partial x_{i}}{\partial q_{i}} \dot{q}_{i}
$$

The force produced by the hydraulic actuator can be calculated as (20), which time derivative is:

$$
\dot{F}_{i}=\dot{P}_{A, i} A_{A, i}-\dot{P}_{B, i} A_{B, i} .
$$

Substituting (23) into (26) allows to define that:

$$
\dot{F}_{i}=\beta \dot{x}_{p, i}\left(\frac{A_{A, i}^{2}}{V_{A, i}}+\frac{A_{B, i}^{2}}{V_{B, i}}\right)+\beta\left(\frac{A_{A, i}}{V_{A, i}} Q_{A, i}+\frac{A_{B, i}}{V_{B, i}} Q_{B, i}\right),
$$

where

$$
\begin{aligned}
V_{A, i} & =\left(V_{h_{A}, i}+A_{A, i} x_{p, i}\right), \\
V_{B, i} & =\left(V_{h_{B}, i}+A_{B, i}\left(s_{i}-x_{p, i}\right)\right) .
\end{aligned}
$$

${ }^{3}$ The telescopic link $q_{4}$ is directly proportional to its piston's displacement. 
Furthermore, replacing (21)-(22) into (27) defines the forces according to the direction of motion:

$$
\begin{aligned}
& \dot{F}_{i}^{+}=-\beta \dot{x}_{p, i}\left(\frac{A_{B, i}{ }^{2}}{V_{B, i}}+\frac{A_{A, i}{ }^{2}}{V_{A, i}}\right)+\beta \psi_{1}\left(u_{i}, P_{A, i}, P_{B, i}\right), \\
& \dot{F}_{i}^{-}=-\beta \dot{x}_{p, i}\left(\frac{A_{B, i}{ }^{2}}{V_{B, i}}+\frac{A_{A, i}{ }^{2}}{V_{A, i}}\right)+\beta \psi_{2}\left(u_{i}, P_{A, i}, P_{A, i}\right),
\end{aligned}
$$

where $(\cdot)^{ \pm}$represents positive or negative input signal, and

$$
\begin{aligned}
& \psi_{1}=\left(\frac{c_{1} u_{i} \sqrt{P_{s}-P_{A, i}} A_{A, i}}{V_{A, i}}+\frac{c_{2} u_{i} \sqrt{P_{B, i}-P_{r}} A_{B, i}}{V_{B, i}}\right) \\
& \psi_{2}=\left(\frac{c_{3} u_{i} \sqrt{P_{A, i}-P_{r}} A_{A, i}}{V_{A, i}}+\frac{c_{4} u_{i} \sqrt{P_{s}-P_{B, i}} A_{B, i}}{V_{B, i}}\right)
\end{aligned}
$$

such that, the force can be denoted in a compact form as:

$$
\dot{F}_{i}=\dot{F}_{i}^{+} \underbrace{\left(\frac{1+\operatorname{sign}\left(u_{i}\right)}{2}\right)}_{u_{p}}+\dot{F}_{i}^{-} \underbrace{\left(\frac{1-\operatorname{sign}\left(u_{i}\right)}{2}\right)}_{u_{n}} .
$$

To define (28) in the linear form:

$$
A \cdot X=\Upsilon \text {, }
$$

we can represent the parameter vector:

$$
X=\left[\begin{array}{lllll}
1 / \beta & c_{1} & c_{2} & c_{3} & c_{4}
\end{array}\right]^{T},
$$

and consider that,

$$
\begin{aligned}
z_{1, i}(t) & =-\frac{A_{A, i} u_{i}(t) \sqrt{P_{s}-P_{A, i}(t)}}{\left(V_{h_{A}, i}+A_{A, i} x_{p, i}(t)\right)}, \\
z_{2, i}(t) & =-\frac{A_{B, i} u_{i}(t) \sqrt{P_{B, i}(t)-P_{r}}}{\left(V_{h_{B}, i}+A_{B, i}\left(s_{i}-x_{p, i}(t)\right)\right)}, \\
z_{3, i}(t) & =-\frac{A_{A, i} u_{i}(t) \sqrt{P_{A, i}(t)-P_{r}}}{\left(V_{h_{A}, i}+A_{A, i} x_{p, i}(t)\right)}, \\
z_{4, i}(t) & =-\frac{A_{B, i} u_{i}(t) \sqrt{P_{s}-P_{B, i}(t)}}{\left(V_{h_{B}, i}+A_{B, i}\left(s_{i}-x_{p, i}(t)\right)\right)},
\end{aligned}
$$

to define the regression matrix:

$A=\left[\begin{array}{lllll}\dot{F}_{i}(t) & z_{1, i}(t) u_{p} & z_{2, i}(t) u_{p} & z_{3, i}(t) u_{n} & z_{4, i}(t) u_{n}\end{array}\right]$,

and the observation vector as:

$$
\Upsilon=-\dot{x}_{p, i}\left(\frac{A_{B, i}{ }^{2}}{V_{B, i}\left(x_{p}(t)\right)}+\frac{A_{A, i}{ }^{2}}{V_{A, i}\left(x_{p, i}(t)\right)}\right) .
$$

\section{ESTIMATION OF MODEL PARAMETERS}

Recalling that dynamics of the robot, as well as the hydraulic system, can be linearly written in the form

$$
\Phi \cdot \Theta=\Sigma,
$$

and that measurements are recorded at each $t_{i}$, with $i=$ $\{1, \ldots, T\}$, an overdetermined matrix of the form

$$
\left[\begin{array}{c}
\Phi\left(t_{1}\right) \\
\Phi\left(t_{2}\right) \\
\vdots \\
\Phi(T)
\end{array}\right] \cdot \Theta=\left[\begin{array}{c}
\Sigma\left(t_{1}\right) \\
\Sigma\left(t_{2}\right) \\
\vdots \\
\Sigma(T)
\end{array}\right]
$$

can be formed for finding an estimate $\hat{\Theta}$ of $\Theta$ that fits the model (34). There are various mathematical forms to define this concept, such as the least-square estimate, which is conceptually the value of $\Theta$ that minimizes the residual of the vector $|\Sigma-\Phi \Theta|$. This can be formulated as

$$
\min \|\Sigma-\Phi \Theta\|^{2} \text {. }
$$

The classical least-square estimate has the unique solution given by

$$
\hat{\Theta}=\left(\Phi^{T} \Phi\right)^{-1} \Phi^{T} \Sigma=\Phi^{\dagger} \Sigma,
$$

where $\left(\Phi^{T} \Phi\right)^{-1} \Phi=\Phi^{\dagger}$ is known as the pseudo-inverse of $\Phi$.

\section{A. Recording data and averaging}

In the machine, different trajectories can be realized by decentralized PD feedback gains [17], i.e.

$$
u_{i}=-K_{p}\left(q_{i}-q_{i}^{r e f}\right)-K_{d}\left(\dot{q}_{i}-\dot{q}_{i}^{r e f}\right)+F_{f}\left(\dot{q}_{i}\right),
$$

where $K_{p}$ denotes the values of proportional gains, $K_{d}$ the values of derivative gains, and $F_{f}$ is a feedforward term used for avoiding dead-zones present in the hydraulic system. The control signal $u_{i}$ is the electrical input level to the electrohydraulic servo valve [17], and the estimation of velocities and accelerations is done by the use of Kalman filtering, as suggested in [24]. The reference trajectories are designed to be periodic, and the data is recorded for various periods, so that it can be averaged to improve the signal-to-noise ratio.

\section{B. Estimation and validation of parameters from (17)}

Various trajectories were recorded to evaluate different ranges of motion. Considering (36), a set of parameters $\left[\hat{\theta}, \hat{\theta}_{f}\right]^{T}$ was found. Some additional data sets were recorded for validation tests, two of which are presented in Fig. $4-5$. The simulation results are shown in Fig. 6 - 7. The validation shows a comparison of the averaged measured torque $\bar{\tau}_{m}$, versus the torque computed by the model (17), once the values of the unknown parameters have been estimated. We can see that major dynamics are successfully recovered by the model, with minor uncertainty not captured due to unmodeled dynamics.

\section{Estimation and validation of parameters (30)}

For simplicity, we present results for the first link, i.e. $q_{2}$. The data used for estimation is presented in Fig. 8. In this figure, the first column shows three different reference trajectories $q_{2}^{r e f}(t)$, at different frequencies. The second column shows the control input (37), while the estimation of (26) is shown in the third column. Applying the set of estimated parameters (30), a comparison of the left hand side of (29), with its right hand side is shown in Fig. 9. These results show agreement between the recorded and the estimated responses of the hydraulic model. 

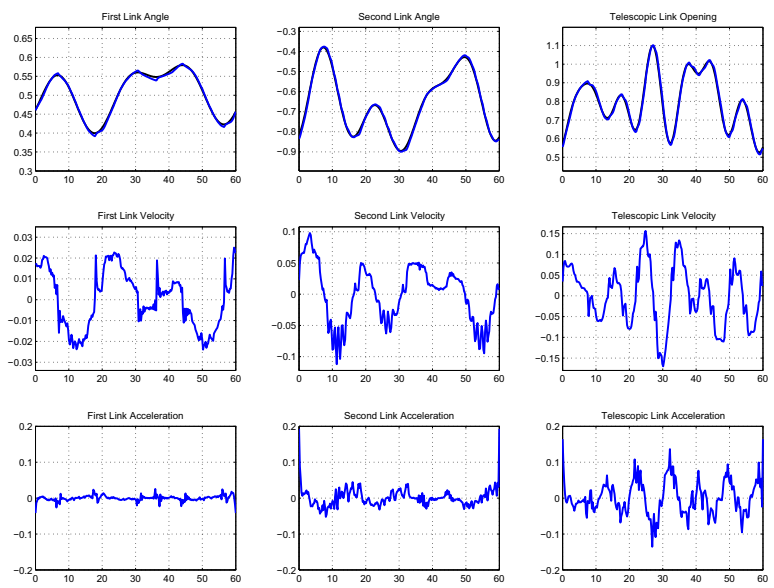

Fig. 4. First example of data used for validation. First row: Averag trajectories in [rad]. Second row: Averaged velocity in [rad/sec]. Third row: Estimated acceleration in $\left[\mathrm{rad} / \mathrm{sec}^{\wedge} 2\right]$. The $\mathrm{x}$-axis is time in seconds.
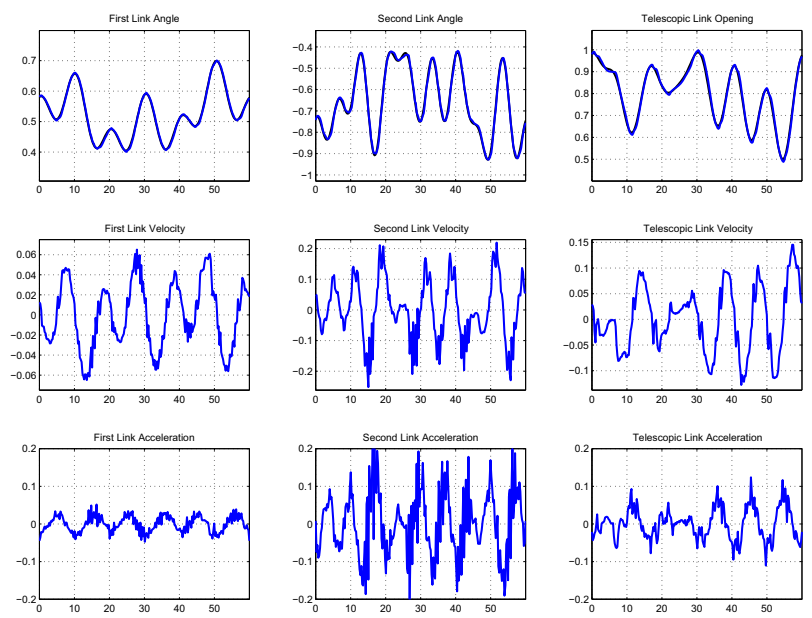

Fig. 5. Second example of data used for validation. First row: Averag trajectories in [rad]. Second row: Averaged velocity in [rad/sec]. Third ro Estimated acceleration in $\left[\mathrm{rad} / \mathrm{sec}^{\wedge} 2\right]$. The $\mathrm{x}$-axis is time in seconds.

\section{CONCLUSions}

We have presented results on modeling and parameters estimation, which were successfully carried on a manipulator used in forestry machines. It is shown that despite the complex interaction between the hydraulic and mechanical processes of the robot, the system's dynamical response can be described by first principle laws. To model friction forces we have considered a map consisting of Coulomb and viscous friction, which are relevant to describe nonlinear dynamics near zero velocities. The geometry of the machine is used to explicitly map the forces of the cylinders to torques on the joints, and to compute their linear velocities given measurements of angular velocities.

To calibrate the model, and to find reliable approximations of unknown parameters, we apply the conventional leastsquare method. To this end, we proposed a transformation
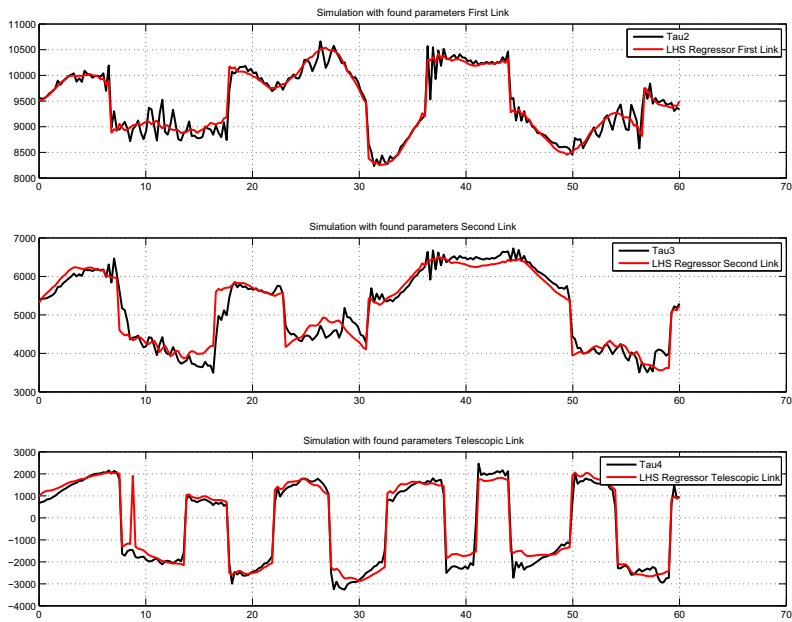

Fig. 6. Validation with the first data set from Fig. 4. The plot shows the measured averaged torque (black line) vs. estimated torque (red line), where the two graphs on top are in $[\mathrm{Nm}]$, and the bottom graph is in $[\mathrm{N}]$. The $\mathrm{x}$-axis is time in seconds.
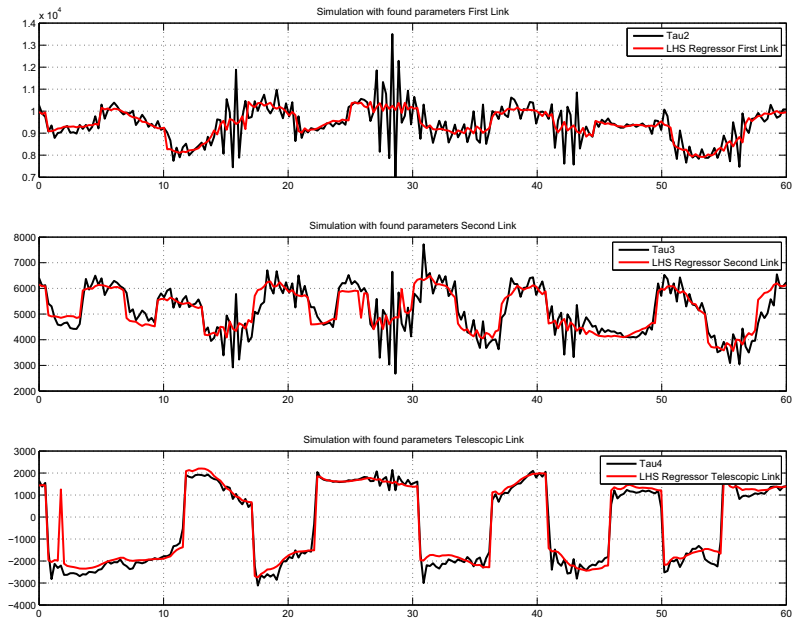

Fig. 7. Validation with the second data set from Fig. 5. The plot shows the measured averaged torque (black line) vs. estimated torque (red line) where the two graphs on top are in $[\mathrm{Nm}]$, and the bottom graph is in $[\mathrm{N}]$. The $\mathrm{x}$-axis is time in seconds.

of the system dynamics into a linear form, possible due to the linearity of the model parameters. To capture the data, we apply decentralized PD control, which has been designed only for the purpose of system identification.

The results allow to assess the correctness of the estimated parameters, and let us conclude that despite minor differences, the model found is able to capture the most relevant dynamics involved in a motion. This statement is valid for the mechanics, as well as for the hydraulic actuation system.

\section{REFERENCES}

[1] B. Löfgren, "Automation of forestry machines - an important piece in precision forestry," in Proceedings of the International Precision Forestry Symposium, (Stellenbosch University, South Africa), 2006. 

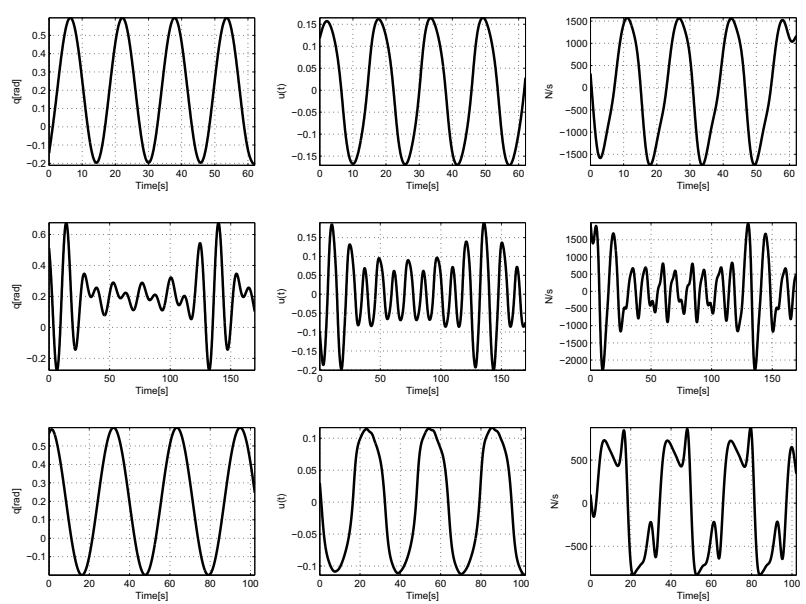

Fig. 8. The data used for estimation. First column: Reference trajectories $q_{2}^{r e f}(t)[\mathrm{rad}]$. Second column: Control signal $u_{2}(t)$. Third column: Estimated derivatives of force $\dot{F}_{2}$. The X-axis is time in seconds.
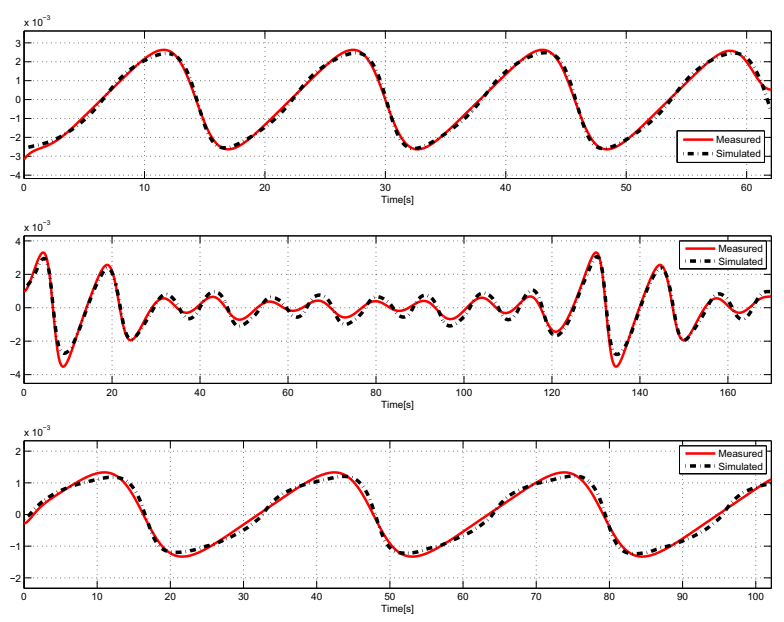

Fig. 9. Validation results: Plot shows the recorded responses (red line) vs. the estimated responses (black line). The $\mathrm{x}$-axis is time in seconds.

[2] U. Hallonborg, "Förarlösa skogsmaskiner kan bli lönsamma (unmanned forestry machines can be competitive)." Skogforsk Results, No 9, 2003.

[3] M. Brander, D. Eriksson, and B. Löfgren, "Automation of knuckleboom work can increase productivity." Skogforsk Results, No 4, 2004.

[4] "Cranab presents an entirely new generation of cranes," June 2011.

[5] A. Shiriaev, L. Freidovich, I. Manchester, U. Mettin, P. L. Hera, and S. Westerbeg, "Status of Smart Crane Lab project: modeling and control for a forwarder crane." Technical report, Department of Applied Physics and Electronics, Umeå University, 2008.

[6] M. Münzer, Resolved Motion Control of Mobile Hydraulic Cranes. $\mathrm{PhD}$ thesis, Aalborg University, 2002.

[7] N. P. Parchuru and J. Thati, "DSP implementation of a control algorithm for a forwarder crane," Master's thesis, Blekinge Institute of Technology, 2010.

[8] M. Linjama, The Modelling and Actuator Space Control of Flexibile Hydraulic Cranes. PhD thesis, Tampere University of Technology, 1998.

[9] S. Rodermond, "Modeling, identification and control of a multivariable hydraulic servo system." Master Thesis, 2006.
[10] R. V. M. Donath, "Dynamic feedback linearization for electrohydraulically actuated servosystem," in Proceedings of the 1992 Japan/USA Symposium on Flexible Automation, (San Francisco, USA), pp. 595606, July 1992.

[11] B. F. and B. Yao, "Adaptive robust precision motion control of singlerod hydraulic actuators with time varying unknown inertia: a case study," in ASME International Mechanical Engineering Congress and Exposition, (Nashville, Tennessee), pp. 131-138, November 15 - 16 1999.

[12] P. L. Hera, U. Mettin, S. Westerberg, and A. Shiriaev, "Modeling and control of hydraulic rotary actuators used in forestry cranes," in Proceedings of the 2009 IEEE International Conference on Robotics and Automation, (Kobe, Japan), pp. 1315-1320, May 12-17 2009.

[13] N. D. Manring, Hydraulic Control Systems. New York, USA: John Wiley \& Sons, first ed., 2005.

[14] M. Jelali and A. Kroll, Hydraulic Servo-Systems: Modeling, Identification and Control. Great Britain: Springer, second ed., 2004.

[15] U. Mettin and P. L. Hera, "Modeling and control design for a hydraulic forestry crane." Research Results, Department of Applied Physics and Electronics, Umeå University, 2005.

[16] P. Lawrence, F. Sassani, B. Sauder, N. Sepehri, U. Wallersteiner, and J. Wilson, "Computer-assisted control of excavator-based machines," in International Off-Highway and Powerplant Congress and Exposition, (Milwaukee, Wisconsin, USA), p. Document number 932486 September 1993.

[17] P. La Hera, U. Mettin, I. Manchester, and A. Shiriaev, "Identification and control of a hydraulic forestry crane," in Proceedings of the 17th IFAC World Congress, (Seoul, Korea), pp. 2306-2311, July 6-11 2008.

[18] J. Mattila and T. Virvalo, "Computed force control of hydraulic manipulators," in 5th Scandinavian Inter. Conf. On Fluid Power, (Sweden), p. 139154, July 6-11 1997.

[19] M. Spong, S. Hutchinson, and M. Vidyasagar, Robot Modeling and Control. New Jersey: John Wiley and Sons, 2006.

[20] P. L. Hera and O. M. D. "Modeling dynamics of an electro-hydraulic servo actuated manipulator: A case study of a forestry forwarder crane," in Proceedings of the World Automation Congress, (Pto. Vallarta, Mexico), 2012.

[21] J. J. Craig, Introduction to Robotics: Mechanics and Control. USA: Prentice Hall, 2003.

[22] L. N. Hand and J. D. Finch, Analytical mechanics. USA: Cambridge University Press, 1998.

[23] H. Olsson, K. Åström, C. Canudas de Wit, M. Gäfvert, and P. Lischinsky, "Friction models and friction compensation," European Journal of Control, vol. 9, no. 4, pp. 176-195, 1998.

[24] P. Belanger, P. Dobrovolny, A. Helmy, and X. Zhang, "Estimation of angular velocity and acceleration from shaft-encoder measurements," The International Journal of Robotics Research, vol. 17, no. 11, pp. 1225-1233, 1998. 